\title{
SEGURIDAD EN LA IDENTIFICACIÓN CORRECTA DEL PACIENTE CRÍTICO
}

\author{
PROPER IDENTIFICATION SAFETY OF CRITICAL PATIENT \\ Javier Rubén Tovar Brandán ${ }^{1 a}$, Teresa Luisa Pareja Pera ${ }^{2 b}$, Javier Rolando Tovar Brandán ${ }^{3 c}$, \\ Michael Jefferson Zubiat Aguilar ${ }^{4 \mathrm{~d}}$
}

\begin{abstract}
RESUMEN
Objetivo: Determinar si existe una identificación adecuada en la recepción, hospitalización y clasificación asistencial en los servicios críticos de Emergencia, Cuidados Intermedios y Cuidados Intensivos de hospitales nacionales (Redes Asistenciales) de EsSalud. Método: Se realizó un muestreo aleatorio recogiendo datos relacionados con el paciente y el sistema de identificación utilizado en los servicios críticos de los Hospitales Nacionales Guillermo Almenara Irigoyen, Edgardo Rebagliati Martins y Alberto Sabogal Sologuren. Resultados: Se incluyeron un total de 632 camas de cuidados críticos. La mayoría de los pacientes son identificados en el $100 \%$ de los casos por nombre en forma verbal, mientras que el número de cama o el cartel en la cabecera es el segundo sistema más usado. Conclusión: Los profesionales encargados de realizar la recepción, hospitalización y clasificación de pacientes, realizan una identificación funcional sin una evidencia tangible directa con el paciente, con lo cual aumenta el riesgo de error y disminuye la seguridad en el paciente ante los procedimientos o diagnósticos.
\end{abstract}

Palabras claves: Sistemas de Identificación de Paciente; Seguridad del Paciente.

\section{ABSTRACT}

Objective: Determine if there is proper identification at the reception, hospitalization and classification in critical care services of Emergency, Care Intermediate and Intensive Care of national hospitals (Care Networks) of EsSalud. Method: A random sampling collecting data related to the patient and the identification system used in critical services of the Hospitales Nacionales Guillermo Almenara Irigoyen, Edgardo Rebagliati Martins and Alberto Sabogal Sologuren, was performed. Results: A total of 632 critical care beds included. Most patients are identified in $100 \%$ of cases by name verbally, while the number of bed or poster at the head is the second most used system. Conclusion: The professionals in charge of reception, hospitalization and patient classification, perform a functional identification without direct tangible evidence with the patient, thereby increasing the risk of error and decreases safety in the patient before or diagnostic procedures.

Key words: Patient Identification Systems; Patient Safety.

\footnotetext{
${ }^{\text {la }}$ Profesor Principal, Departamento de Ciencias Dinámicas, Facultad de Medicina San Fernando, Universidad Nacional Mayor de San Marcos (Lima - Perú)

${ }^{2 a}$ Profesor Auxiliar, Departamento de Enfermería, Facultad de Medicina San Fernando, Universidad Nacional Mayor de San Marcos (Lima - Perú)

${ }^{3 c}$ Jefe de Servicio Anestesiología, Hospital Nacional Edgardo Rebagliati Martins - EsSalud (Lima - Perú)

${ }^{4 d}$ Jefe de la Oficina de Secretaria Técnica, Red Asistencial Lambayeque - EsSalud (Lima - Perú)
} 


\section{INTRODUCCIÓN:}

Los profesionales del sector salud suelen despersonalizar a los pacientes y es frecuente que se refieran a ellos por el número de cama o por su enfermedad y no por su nombre. Los errores ocasionados por este tema son la segunda causa de error médico en el medio hospitalario, por lo que se requiere tomar medidas y realizar una autocrítica en relación a la metodología utilizada en la actualidad. La informática y las técnicas de la Organización Mundial de la Salud (OMS) aportan elementos de suma importancia para minimizar este problema.

La forma más habitual de identificación de los pacientes en centros hospitalarios es el número de habitación o de cama; el diagnostico; las características físicas o psicológicas; o el hecho de que respondan a un nombre determinado. Sin embargo, los problemas relacionados con el estado cognitivo, el lenguaje, la audición, el idioma o la edad de las personas hospitalizadas se pueden convertir en barreras que pueden dificultar la seguridad de la identidad.

La seguridad del paciente ha adquirido una gran importancia en los últimos años como expresión de una atención sanitaria de calidad, siendo la aspiración tanto de pacientes ${ }^{1}$ como de profesionales sanitarios ${ }^{2}$ ,cuyo objetivo no es otro que prestar una asistencia sanitaria segura, efectiva y eficiente. La principal razón para este interés se encuentra en el aumento de la complejidad de los pacientes que se tratan y de los sistemas sanitarios. Todo esto conlleva que los daños que se puedan ocasionar tengan una importancia considerable tanto desde el punto de vista humano como económico ${ }^{3}$.

Organizaciones de la importancia de la $\mathrm{OMS}^{4}$ y la Organización Panamericana de la Salud han desarrollado estrategias para intentar controlar estos eventos. Dentro del ámbito iberoamericano, el Ministerio de Salud de España ${ }^{5}$ ha situado la seguridad del paciente como uno de los elementos claves, desarrollándola dentro de sus estrategias (estrategia número 8), lo que se refleja en el Plan de Calidad para el Sistema Nacional de Salud ${ }^{6}$.

La perspectiva de los ciudadanos por la seguridad del paciente ha sido analizada por diferentes estudios, llegándose a la conclusión de que es un tema de gran impacto en la población $n^{7}$. Los pacientes desean sentirse seguros y confiados con los cuidados sanitarios recibidos. Por tanto, la seguridad en los procedimientos a realizar se plantea como un reto para los encargados de la atención de cara a los usuarios.

Se realiza el presente trabajo para determinar si existe una identificación adecuada en la recepción, hospitalización y clasificación asistencial en los servicios críticos de Emergencia, Cuidados Intermedios y Cuidados Intensivos de Hospitales Nacionales (Redes Asistenciales) de EsSalud.

\section{MATERIALES Y MÉTODOS}

El presente es un estudio de tipo observacional y descriptivo realizado en agosto del 2014 en los en los servicios críticos de los Hospitales Nacionales Guillermo Almenara Irigoyen, Edgardo Rebagliati Martins y Alberto Sabogal Sologuren. Se excluyeron los pacientes hospitalizados en el servicio de neonatología.

Se realizó un muestreo aleatorio sin reemplazo, recogiendo datos relacionados con el paciente y el sistema de identificación utilizado. Dado que la seguridad en la identificación del paciente en el proceso de recepción, hospitalización y clasificación es funcional, se recopiló información que no es tangible en relación a la identidad e identificación del paciente.

La información se recolectó en formatos diseñados especialmente para el trabajo. La presentación de los resultados se realizó mediante el uso de tablas y gráficos. Los cálculos estadísticos fueron realizados usando el software SPSS.

\section{RESULTADOS:}

Se incluyeron un total de 632 camas de cuidados críticos ocupadas al $100 \%$, cuya distribución por hospital y servicio crítico se aprecia en la Tabla 1.

\begin{tabular}{lcccc}
\hline Tabla 1. Número de camas según hospital y servicio crítico \\
\hline Establecimiento & \multicolumn{5}{c}{ Número de camas } \\
\cline { 2 - 5 } & EMG & UCl & UCIN & Total \\
Hospital Guillermo Almenara & 73 & 57 & 73 & 203 \\
Hospital Edgardo Rebagliati & 122 & 104 & 109 & 335 \\
Hospital Alberto Sabogal & 29 & 30 & 35 & 94 \\
\hline Total & 224 & 191 & 217 & 632
\end{tabular}

Fuente: Gerencia Central de Prestaciones de Salud

La mayoría de los pacientes son identificados por el personal asistencial al $100 \%$ por nombre en forma verbal, lo cual sólo es posible cuando el paciente no tiene compromiso de conciencia, mientras que el número de cama o el cartel en la cabecera es el segundo sistema más usado (figura 1).

Figura 1. Sistema de identificación del paciente en las áreas críticas de los Hospitales Almenara, Rebagliati y Sabogal

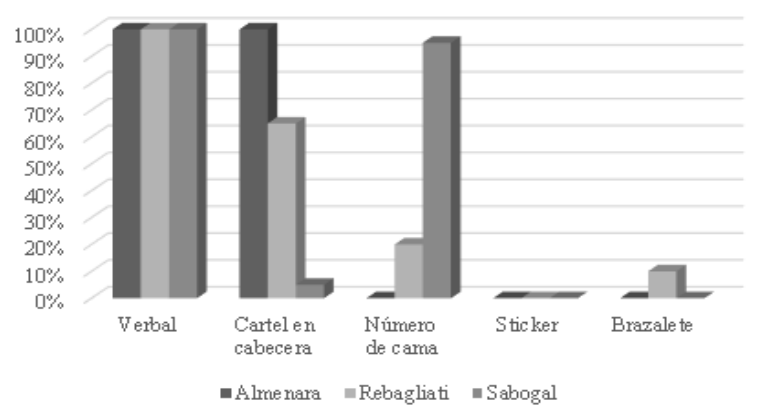

Se conserva el sistema actual de identificación del paciente en los hospitales Almenara y Sabogal, destacando el sistema de pulsera como opción en el servicio de emergencia del Hospital Rebagliati (figura 2). 
Figura 2. Sistema de identificación tradicional e innovadora en las áreas críticas de los Hospitales Almenara. Rebaaliati v Saboaal.

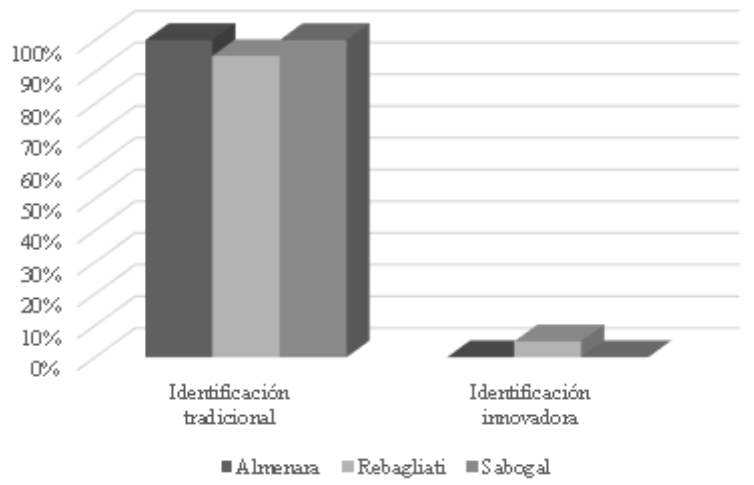

Nınguno de los protesıonales de entermeria ıntorma al paciente la razón por la que se le coloca sus datos de filiación en un cartel, un sticker adherido a su ropa, u otro medio. El $99 \%$ no explica el procedimiento al paciente, sin tener en cuenta que el usuario no comprende la importancia de los medios de identificación.

\section{DISCUSIÓN:}

La causa más frecuente de problema para identificar al paciente por parte de la enfermera que realiza la recepción, hospitalización y clasificación de enfermos, son los pacientes que entran directamente al área de cuidados críticos. Esto se debe a que el ingreso de estos pacientes se hace por una vía que no es la habitual, como consecuencia del carácter emergente de la atención que precisan. Por ello, es necesario una atención más detallada entre los profesionales de enfermería con este tipo de pacientes.

El establecimiento de protocolos en los servicios clínicos y quirúrgicos, y con mayor relevancia en los cuidados críticos, es la herramienta clave para una gestión asistencial, eficiente, eficaz y que garantiza la seguridad clínica del paciente. El seguimiento del protocolo establecido es la base para una actuación de calidad, pero esto en nuestra institución no existe: la identificación es funcional y no tangible, lo que la convierte en un sistema que no es correcto ni seguro en la identificación del paciente.

Existe un importante grupo de profesionales que ven de forma positiva cambiar el sistema tradicional funcional convencional de identificación por un sistema tangible relacionado directamente con el paciente, a fin de evitar la retirada o cambio accidental de la misma así como otros errores de una identificación inadecuada. El consenso de los expertos e informes evidencian una considerable reducción de errores en establecimientos individuales luego de la implementación de procesos revisados de identificación de pacientes en el internamiento, hospitalización y, fundamentalmente, en áreas críticas. La mayoría de estos últimos está imposibilitado de una comunicación eficiente y efectiva ya sea por sedación, trauma encefálico u otras patologías del sensorio.

Entre los sistemas de identificación existentes, el que sería más adecuado para las características de nuestro sistema de salud es el brazalete de identificación (BI). Este consiste en una pulsera de polipropileno con un área imprimible y un cierre seguro e inviolable que garantiza que el brazalete no se abra durante la estadía del paciente (figura 3). Este sistema se viene usando en el Departamento de Emergencia del Hospital Edgardo Rebagliati Martins en forma parcial y no oficial, habiendo aumentado la seguridad en los tratamientos médicos, quirúrgicos y farmacológicos. Se trata de una medida que ha permitido aumentar la calidad de la atención brindada por nuestra institución.

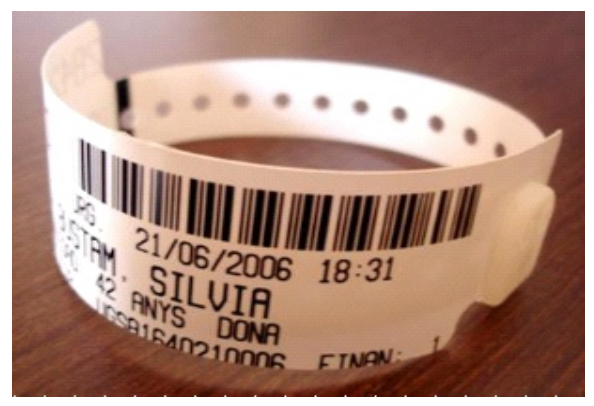

Figura 3. Brazalete de identificación

Es necesario reforzar entre los profesionales de salud la necesidad de registrar por escrito, además de la identificación, las alergias que sufre el paciente. Sólo los registros escritos son una garantía de calidad para la seguridad del paciente. 8 Estos deben realizarse no sólo en la historia clínica, sino también colocando sistemas de identificación visuales como la pulsera de color fluorescente u otra alerta con el tipo de alergia, lo que no observamos en ninguno de los servicios críticos incluidos en el presente estudio.

La evidencia en el presente trabajo no permite afirmar que exista o hayan existido errores en la metodología de la identificación de los pacientes como consecuencia de la forma como se maneja actualmente la identificación en EsSalud, el que tiene las siguientes características:

El número de habitación y/o cama es lo que marca la pauta en todos los servicios del hospital.

La mayoría del personal de las áreas involucradas en el proceso no cumple con los estándares operacionales y muchos de ellos no los conocen.

Es difícil crear una cultura de cambio en las personas debido a que las actividades se realizan en forma rutinaria.

No se cuenta con un equipo de trabajo comprometido y dispuesto a asumir las responsabilidades que se requieran en el proyecto de implementación de un nuevo sistema de identificación.

No existe educación a los pacientes sobre los riesgos relacionados con la identificación incorrecta 
de los mismos.

No se pide a los pacientes o a sus familiares que verifiquen la información de identificación para confirmar que sea correcta.

No se solicita a los pacientes que se identifiquen antes de recibir cualquier medicamento y previo a cada diagnóstico o intervención terapéutica.

A pesar que los neonatos tienen $\mathrm{BI}$, las madres no son instruidas en la verificación de la identificación del recién nacido durante el post parto, lactancia y puerperio.

\section{CONCLUSIONES:}

Los profesionales encargados de realizar la recepción, hospitalización y clasificación de pacientes, realizan una identificación funcional sin una evidencia tangible directa con el paciente, con lo cual aumenta el riesgo de error y disminuye la seguridad en el paciente ante los procedimientos 0 diagnósticos.

En tal sentido, la administración debe disponer la implantación de los recursos materiales de identificación diferenciado para los pacientes, sugiriendo el uso del $\mathrm{BI}$ con registro Informático.

\section{CONFLICTOS DE INTERÉS}

Los autores declaran no tener conflictos de interés en la publicación de este artículo.

\section{REFERENCIAS BIBLIOGRÁFICAS}

1. Ministerio de Sanidad, Política Social e Igualdad. La perspectiva de los ciudadanos por la seguridad del paciente. Madrid: Ministerio de Sanidad, Política Social e Igualdad; 2011.

2. Ministerio de Sanidad y Política Social. Estándares de calidad de cuidados para la seguridad del paciente en los hospitales del SNS. Proyecto SENECA: informe técnico 2008. Madrid: Ministerio de Sanidad, Política Social Igualdad; 2010.

3. Aranaz-Andrés JM, Limón-Ramírez R, AibarRemón C, Miralles-Bueno JJ, Vitaller-Burillo J, TerolGarcía E, et al. Luces y sombras en la seguridad del paciente: estudio y desarrollo de estrategias. Informe SESPAS 2008. Gac Sanit. 2008;2 Supl 1:198-204.

4. Organización Mundial de la Salud. Alianza mundial para la seguridad del paciente. La investigación en seguridad del paciente: mayor conocimiento para una atención más segura. Ginebra: Organización Mundial de la Salud; 2008.

5. Convenio de colaboración entre el Ministerio de Sanidad y Consumo y la Comunidad Autónoma de Andalucía para el impulso de prácticas seguras en los centros sanitarios. Resolución 1604 de la Secretaría General de Sanidad. Boletín Oficial del Estado Núm 22 del 25 ene 2007.

6. Presidencia Española de la Unión Europea. V Conferencia Internacional de Seguridad del Paciente. Infección relacionada con la atención sanitaria y resistencia antimicrobiana. Madrid 3-4 jun 2010;
Madrid: Ministerio de Sanidad y Política Social; 2010.

7. Sociedad Española de Enfermería de Urgencias y Emergencias. Recepción, acogida y clasificación asistencial de los pacientes en urgencias: recomendación científica 99/01/01, de 15 de junio de 1999. Disponible en: http://www.enfermeriadeurgencias.com/documentos/ recomendaciones/REVISIONRAC.pdf.

8. McCloskey J, Bulechek GM, editores. Clasificación de intervenciones de enfermería (NIC). $4^{a}$ edición. Madrid: Elsevier España; 2005. 